Originalveröffentlichung in: O. Hekster, S. Schmidt-Hofner, Chr. Witschel (Eds.), Ritual Dynamics and Religious Change in the Roman Empire. Proceedings of the Eighth Workshop of the International Network Impact of Empire (Heidelberg, July 5-6, 2007), Impact of Empire 9, Leiden/Boston 2009, S. $349-366$

\title{
MINIATURISIERUNG ALS SCHLÜSSEL ZUM VERSTÄNDNIS RÖMERZEITLICHER ÄGYPTISCHER RITUALE?
}

\section{Joachim-Friedrich Quack}

In den nachfolgenden Ausführungen wird es schwerpunktmäßig um eine Auseinandersetzung mit bestimmten Forschungspositionen hinsichtlich magischer Rituale aus dem spätantiken Ägypten gehen. Das relevante Quellenmaterial besteht vor allem aus Papyri in griechischer, seltener auch ägyptischer (demotischer) Sprache, die in der Mehrzahl aus der späteren Römerzeit, also dem 3. und 4. Jahrhundert n. Chr. stammen. Ein erheblicher Teil davon ist von Karl PREISENDANZ in seiner maßgeblichen Edition als Papyri Graecae Magicae zusammengestellt worden; ${ }^{1}$ anderes Material ist inzwischen ebenfalls, vor allem in der Publikation Supplementum Magicum, zusammenhängend greifbar. ${ }^{2}$ Diese Handschriften enthalten zahlreiche Anweisungen für die praktische Durchführung von Ritualen, ja sie stellen für den griechischsprachigen Bereich mutmaßlich das umfangreichste und detaillierteste Corpus dieser Art überhaupt dar. Allerdings sollte man sich davor hüten, sie aufgrund der dominierenden Sprachform einfach als Quellen für die griechische Kultur anzusehen; vielmehr sind ägyptische und auch jüdische Einflüsse in erheblichem Umfang greifbar.

Jonathan Z. SMITH nimmt als Ausgangspunkt seiner Untersuchungen die These, dass in der Spätantike in einer Reihe von religiösen Traditionen Tempelopfer, insbesondere solche, die mit Tieropfern zu tun hatten, außer Gebrauch gekommen seien. ${ }^{3}$ Teilweise sei-

${ }^{1}$ K. Preisendanz, Papyri Graecae Magicae. Die griechischen Zauberpapyri (Leipzig - Berlin 1928-31; Stuttgart 1973-74, 2. Aufl.).

${ }^{2}$ R.W. Daniel - F. Maltomini, Supplementum Magicum I-II (Opladen 1990/92). Englische Übersetzung: H.D. Betz (ed.), The Greek Magical Papyri in Translation Including the Demotic Spells (Chicago - London 1986). Überblick mit sehr umfangreicher Literatur: W. Brashear, 'The Greek magical papyri: an introduction and survey; annotated bibliography (1928-1994)', in: Aufstieg und Niedergang der römischen Welt II 18, 5 (Berlin - New York 1995), 3380-3684.

${ }^{3}$ J.Z. Smith, Map is not Territory. Studies in the History of Religions (Leiden 1978; ND Chicago - London 1993), 172-189: “The Temple and the Magician"; vgl. 
en moralische Bedenken der Auslöser dafür gewesen, vom Blutvergießen zu reinen Getreide- und Räucheropfern überzugehen. Besonders in der jüdischen und christlichen Gemeinschaft sei der Akt des Opferns durch Erzählungen und Diskurs ersetzt worden. SMITH sieht die magischen Papyri der Spätantike zusammen mit den theurgischen und alchemistischen Traktaten derselben Zeit als einzige größere Materialgruppe dieser Epoche an, in denen Opferstrukturen und Opferterminologie weiterverwendet worden seien. Neben einer kleineren Zahl von Ritualorten in freiem Gelände sei die häusliche Umgebung vor allem des Magiers selbst (eher als die des Kunden) eindeutig dominant gewesen. Vorzugsweise habe man unblutige Opfer dargebracht, insbesondere Weihrauch. Rein tierische Opfer seien selten gewesen; der einzige Fall der Darbringung eines ganzen Säugetieres beruhe auf einer modernen Konjektur (zu PGM IV 2394-2399). ${ }^{4}$ Gemischt tierisch-pflanzliche Opfer seien zwar häufiger als rein tierische vorgekommen, aber immer noch seltener als die ausschließlich pflanzlichen. Dabei sei zumeist eine Reihe von Pflanzen mit einem tierischen Teil zusammen dargebracht worden. Etliche dieser tierischen Bestandteile wie Kot, Eier oder die Haut einer Schlange hätten im übrigen noch nicht einmal eine Tötung erfordert. Somit stehe insgesamt die Opferung von Tieren eindeutig nicht im Zentrum dieser Textgruppe.

Weiterhin betont SMITH, wie häufig in dieser Textgruppe miniaturisierte Versionen von Ritualen zu finden seien. So gäbe es zum Teil Miniaturaltäre aus nur zwei bis allenfalls sieben Ziegeln, auf denen ebenso kleine Opfer dargebracht würden, teilweise nur ein Samen Sesam oder schwarzer Kümmel, als umfangreichstes Opfer ein Hahn. Ebenso würden häufig kleine Schreine für entsprechend kleine Götterstatuen gebaut. Unter ihnen gäbe es etwa einen mumifizierten Falken, eine Hermesgestalt aus Teig, eine Statuette der Selene, eine drei Handbreit hohe Wachsfigur oder einen auf einem Tisch stehenden Miniatur-Tempel, in den ein kleiner Teller gestellt werde. Diese Anlagen würden aber so gehandhabt, als handele es sich um größere Gebäude, die ein Götterbild mit Kulttisch enthielten. Die besten Pa-

ferner dens., 'Trading places', in: M. Meyer - P. Mirecki (eds.), Ancient Magic and Ritual Power (Leiden 1995), 13-27, bes. 20-27.

${ }^{4}$ Tatsächlich ist zwar an der Stelle die Emendation zu einem Onager eine Konjektur, die Erwähnung der Eingeweide, die geröstet werden, lässt aber an der Opferung eines Tieres an sich keinen Zweifel. 
rallelen hierfür seien in den häuslichen Ritualen in kleinem Maßstab zu suchen, in denen normale Personen Opfer für ihre Hausgötter und/oder Ahnen durchführten. Der wesentliche Unterschied zwischen diesen und den Praktiken der griechischen magischen Papyri bestehe darin, dass letztere aus dem familiären Rahmen herausgerissen und sowohl sehr mobil als auch professionell geworden seien. Die Sakralität eines Platzes würde temporär hergestellt, und zwar durch rituelle Aktivität und die direkte Erfahrung eines mobilen professionellen Ritualisten im Verbund mit einer mobilen Gottheit.

Zudem sei der wichtigste rituelle Akt überhaupt in diesem Corpus weder die Reinigung noch die Inkubation noch das Opfer, sondern der Akt des Schreibens an sich gewesen. Der Glaube an die Wirksamkeit des Schreibens äußere sich in zahlreichen Rezepten zur Herstellung von Amuletten und Phylakteren. Dies sei nicht einfach als Ersatz des archaischen Tempels als Hauptort von Schreibaktivität und Bücherbewahrung zu verstehen, sondern als ein Ersatz der rituellen Praxis durch Schriftgebrauch, analog zu dem des Opfers durch Sprache im Judentum und Christentum. In dem Maße wie das Ritual ohnehin schon eine Miniaturisierung und gleichzeitig Übertreibung von Alltagshandlungen gewesen sei, könnten die griechischen magischen Papyri mit ihren Miniaturisierungen als Ritual eines Rituals betrachtet werden.

Unter Anwendung dieser Analysekategorie der ,Miniaturisierung' haben MOYER und DIELEMAN ein spezifisches Ritual der Textgruppe genauer analysiert. ${ }^{5}$ Dabei handelt es sich um die Weihe eines Ringsteines, in den ein spezielles Bild graviert ist. Der verwendeten Terminologie nach wird er als Kultstatue ( $\xi o ́ \alpha v o v)$ verstanden. Ein bestimmter ritueller Text (der griechisch geschrieben ist, aber sowohl jüdische als auch ägyptische Elemente enthält) muss unter komplexen Bedingungen mehrfach rezitiert werden, und zwar beim Stand des Mondes in bestimmten Tierkreiszeichen. Dabei finden auch Libationen und Weihräucherungen statt. Der Stein wird nicht einfach als Objekt mit magischen Fähigkeiten betrachtet, sondern als lebendes Abbild des Gottes, das in seiner Funktion mit Kultbildern in Tempeln vergleichbar sei.

${ }^{5}$ I.S. Moyer - J. Dieleman, 'Miniaturization and the opening of the mouth in a Greek magical text (PGM XII.270-350)', Journal of Ancient Near Eastern Religions 3 (2003), 47-72. 
Ein Kernpunkt von MOYERs und DiELEMANs Argumentation ist, dass die Weihe und Anrufung des Steines im Text selbst als Ouphôr bezeichnet wird. Dieses Wort kann aber mit hoher Wahrscheinlichkeit als phonetische Wiedergabe des ägyptischen Terminus wpi.t-r' („Mundöffnung“) erkannt werden, welches der Titel eines gut bekannten und ausführlichen ägyptischen Rituals ist. ${ }^{6}$ Dieses Ritual ist primär für Kultstatuen, aber auch für Mumien bezeugt. In seiner Anwendung auf ein so kleines Objekt wie einen Ringstein sehen MOYER und DIELEMAN ein gutes Beispiel eben für die Miniaturisierung. Darüber hinaus betonen sie, dass die magische Praxis sich zwar durch diesen Namen auf ein bekanntes Ritual beziehen ließe, die Beziehung zu dem spezifischen Wortlaut des Rituals jedoch alles andere als klar sei. Unter Berufung auf Levi-Strauss ${ }^{7}$ behaupten sie, bei der Miniaturisierung sei der Verlust an der sinnlich wahrnehmbaren Erscheinungen mit einem Gewinn an der intellektuell erfassbaren Dimension einhergegangen. Als von Menschen gemachte Miniaturisierung würde es sich nicht einfach um passive Projektionen des Objektes selbst handeln, sondern um ein aktives Experimentieren damit.

Man könnte das Phänomen der ,Miniaturisierung' auch noch an einem anderen Befund exemplifizieren, den ich selbst herausgearbeitet habe. ${ }^{8}$ Dort geht es um ein komplexes Ritual der Traumsendung, dessen Handlungsteil insbesondere die Herstellung einer Figurine beinhaltet, die sich klar an den Grundprinzipien eines Korn-Osiris der osirianischen Tempelrituale orientiert. Allerdings ist die Figurine nur sieben Finger groß und nicht sieben Handbreit (eine Elle), wie es das Normmaß der Tempelrituale ist. Die Miniaturisierung wäre hier also hervorragend zu fassen, und zwar ganz konkret dadurch, dass man

${ }^{6}$ Die Standardedition ist E. Otto, Das ägyptische Mundöffnungsritual (Wiesbaden 1960); vgl. dazu zuletzt J.F. Quack, 'Fragmente des Mundöffnungsrituals aus Tebtynis', in: K. Ryholt (ed.), The Carlsberg Papyri 7. Hieratic Texts from the Collection (Kopenhagen 2006), 69-150 mit weiteren Angaben; siehe ferner M. F. Ayad, 'Towards a better understanding of the opening of the mouth ritual', in: J.-C. Goyon - C. Cardin (eds.), Proceedings of the Ninth International Congress of Egyptologists - Actes du neuvième Congrès international des égyptologues, Grenoble 2004 (Leuven - Paris - Dudley/MA 2007), 109-116.

${ }^{7}$ C. Levi-Strauss, The Savage Mind (Letchword 1966), 23-24; im Original ders., La pensée sauvage (Paris 1962), 34-36.

8 J.F. Quack, 'Remarks on Egyptian rituals of dream-sending', in: P. Kousoulis (ed.), Ancient Egyptian Demonology. Studies on the Boundaries between the Divine and the Demonic in Egyptian Magic (Leuven - Paris - Dudley/MA, 2008), 129-150. 
zwar die Zahlen beibehalten, aber diese in der nächst kleineren Maßeinheit berechnet habe.

Um eine solche Behauptung bewerten zu können, muss man jedoch in Betracht ziehen, wie sich die Überlieferungslage insgesamt darstellt und in welchem Maße für die Spätantike eine spezifische Situation zu fassen ist - aber auch, inwiefern insbesondere SMITH das einschlägige Material tatsächlich vollständig präsent war. Der Kernpunkt der Probleme, die ich mit seinem Ansatz habe, ist dabei, dass SMITH vorrangig von der allgemeinen Religionswissenschaft kommt und allenfalls noch das Material in griechischer Sprache angemessen heranzieht. Die Quellen in ägyptischer Sprache und Schrift scheinen ihm dagegen fremd zu sein. Gerade ihre angemessene Heranziehung sehe ich aber als fundamental an, wenn man anhand einer Textgruppe argumentiert, deren Fundort eben das spätantike Ägypten ist, und in der generell - wie allgemein anerkannt wird - so viele ägyptische Elemente vorhanden sind. ${ }^{9}$

Vor dem Hintergrund des ägyptischen Befundes entlarvt sich ein Punkt sofort als irrelevant, dem SMITH große Aufmerksamkeit geschenkt hat, nämlich die Frage nach dem Verhältnis von tierischen zu pflanzlichen Opfern. Für die griechisch-römische Antike, in der es bereits einen innerkulturellen Diskurs darüber gab, ob man Tieropfer durchführen sollte, ${ }^{10}$ mag diese Unterscheidung fundamental gewesen sein. Für Ägypten, wo es keinen überlieferten expliziten Meta-

${ }^{9}$ Eine ausführliche Diskussion über den genauen Anteil ägyptischer Elemente erscheint an dieser Stelle entbehrlich. Während R.K. Ritner, 'Egyptian magical practice under the Roman Empire: the demotic spells and their religious context', in: Aufstieg und Niedergang der römischen Welt II, 18, 5 (Berlin - New York 1995), 3333-3379 ihre Relevanz wohl etwas übertrieben hat, haben verschiedene seiner Kritiker sie zweifellos etwas unterschätzt. Für meine eigene Position verweise ich auf J.F. Quack, 'Kontinuität und Wandel in der spätägyptischen Magie', Studi Epigrafici e Linguistici 15 (1998), 77-94; ders., 'Griechische und andere Dämonen in den demotischen magischen Texten', in: T. Schneider (Hrsg.), Das Ägyptische und die Sprachen Vorderasiens, Nordafrikas und der Ägäis. Akten des Basler Kolloquiums zum ägyptisch-nichtsemitischen Sprachkontakt, Basel 2003 (Münster 2004), 427-507; ders., 'From ritual to magic. Ancient Egyptian forerunners of the Charitesion and their social setting', in: G. Boak - Y. Harari - S. Shaked (eds.), Continuity and Innovation in the Magical Tradition (im Druck).

${ }^{10}$ Als bekanntestes Werk sei hier nur Porphyrios' Abhandlung De Abstinentia genannt. Vgl. generell P. Chuvin, Chronique des derniers païens (Paris 1990), $237-$ 244; G.G. Stroumsa, La fin du sacrifice. Les mutations religieuses de l'antiquité tardive (Paris 2005), 108-114. 
diskurs gab, ${ }^{11}$ Tieropfer nie als Problem angesehen wurden und wo vor allem die Eigenterminologie keinerlei Anlass zu der Annahme gibt, dass überhaupt ein grundlegender Unterschied zwischen tierischen und pflanzlichen Opfern gemacht wurde, ist sie hingegen ohne jede Bedeutung. Jede Betrachtung eines größeren ägyptischen Rituals zeigt sofort, dass es dabei grundsätzlich ein Nebeneinander von tierischen und pflanzlichen Opferbestandteilen gab, und zwar bereits im Rahmen des täglichen Tempelopfers, wo beides in erheblichem Umfang vorkam. ${ }^{12}$ Die Art und Menge der geopferten Tiere dürfte dabei allenfalls eine Frage der materiellen Leistungsfähigkeit der Durchführenden gewesen sein, sobald man sich aus dem Rahmen des staatlichen Opferkultes heraus und in die privateren Rituale hinein bewegte.

Letzteres ist übrigens auch für die Bewertung der griechischen magischen Papyri ein grundlegend wichtiger Punkt, der von SMITH erstaunlicherweise überhaupt nicht diskutiert wird, obwohl er immer wieder von der Professionalität des Ritualisten spricht. Immerhin handelt es sich um ein Corpus von Ritualen, die außerhalb eines öffentlich finanzierten Kultbetriebes ablaufen mussten und auch nicht die Gemeinschaft einer ganzen Siedlung beziehungsweise einer größeren Kultgemeinschaft involvierten, sondern allein einen professionellen Magier und seinen einzelnen Kunden betrafen. ${ }^{13}$ Dies werden aber in der überwiegenden Mehrheit nicht Leute gewesen sein, welche den Kaufpreis für ein ganzes Rind oder gar mehrere problemlos auslegen konnten. Folglich stellte die Reduzierung in der Dimension der Opfer im privaten Bereich auf die Größe allenfalls von Vögeln zuallererst eine schlichte ökonomische Notwendigkeit dar, um überhaupt ein funktionierendes System zu garantieren. Dabei sind die Dimensionen gelegentlich auch nicht ganz so bescheiden, wie SMITH behauptet. Ein Text zur Ringweihe zeigt mit einer Gans, drei Hähnen

${ }^{11}$ Allenfalls - und bezeichnenderweise gerade nicht von seiten eines Ägypters geäußert - kann man die Darlegung bei Plutarch, De Iside, Kap. 31 hierhin stellen, daß in Ägypten ein angemessenes Opfer nicht etwas sei, was die Göttern liebten, sondern vielmehr Tiere, welche Inkarnationen von gottlosen und ungerechten Menschen darstellten.

${ }^{12}$ Hier mag ein Verweis auf W. Barta, Aufbau und Bedeutung der altägyptischen Opferformel (Glückstadt 1968) genügen.

${ }^{13} \mathrm{Vgl}$. auch S.I. Johnston, 'Sacrifice in the Greek magical papyri', in: P. Mirecki / M. Meyer (eds.), Magic and Ritual in the Ancient World (Leiden/Boston 2002), 344-358. 
und drei Tauben schon recht substantielle tierische Opfer, wobei noch Libationen mit Wein, Honig, Milch und Safran hinzukommen (PGM XII 211-216). Ein anderes Ritual erfordert es, gleich sieben verschiedene Vögel (einen Hahn, eine Wachtel, einen Zaunkönig, eine Taube, eine Turteltaube und zwei beliebige Junge) zu erdrosseln (PGM XII 30-31). Insgesamt kann man hier kaum von einer wirklichen Tendenz sprechen, auf blutige Opfer zu verzichten, und die Kombination tierischer und pflanzlicher Substanzen stellte ohnehin keine Neuentwicklung dar.

Weiterhin scheint es relevant, auf die Quellenfrage genauer einzugehen. Generell sind erhaltene Ritualbeschreibungen aus der Antike nicht allzu häufig. Die christlichen Abschreiber der antiken Literatur hatten verständlicherweise keinerlei Interesse daran, solche Textsorten zu tradieren. So können wir allenfalls kurze, mehr oder weniger verzerrte und einseitig ausgedeutete Besprechungen paganer Bräuche im Rahmen apologetischer Traktate christlicher Autoren oder aber Diskurse im Rahmen andersartig gelagerter Werke wie die in Macrobius' Saturnalia heranziehen, wo es darum geht, welche Tieropfer für welche Gottheiten angemessen sind (Saturnalia 3, 112). Angewiesen sind wir somit im wesentlichen auf die epigraphische Überlieferung sowie auf Bodenfunde von Ritualhandschriften. Letztere sind nur dort in größerer Zahl vorhanden, wo die Gegebenheiten der Lagerung und der verwendeten Textträger eine Erhaltung realistisch machen. Konkret betrifft das innerhalb der Alten Welt wohl nur Ägypten mit seinem Wüstenklima, das Papyruserhaltung (zumindest an Orten, die dauerhaft oberhalb des Grundwasserspiegels liegen) ermöglicht, sowie Mesopotamien, wo die Tontafeln mit Keilschrift als Textträger vergleichsweise robust sind. Und tatsächlich liegen aus dem Bereich des Zweistromlandes gerade in Form der Graeco-Babyloniaca bis ans Ende der Keilschriftkultur Ritualtexte vor, die mutmaßlich in ihrer Überlieferung sogar bis in das 3. Jahrhundert n. Chr. reichen. ${ }^{14}$

${ }^{14}$ M.J. Geller, 'The last wedge', Zeitschrift für Assyriologie 87 (1997), 43-95; S. Houston - J. Baines - J. Cooper, 'Last writing: script obsolescence in Egypt, Mesopotamia and Mesoamerica', Comparative Studies in Society and History 45 (2003), 430-479; J. Oelsner, 'Incantations in southern Mesopotamia - from clay tablets to magical bowls (thoughts on the decline of the Babylonian culture)', in: S. Shaked (ed.), Officina Magica. Essays on the Practice of Magic in Antiquity (Leiden - Boston 2005), 31-51. 
Ich werde mich nun aber auf den ägyptischen Befund konzentrieren. Epigraphisch war hier gerade in der griechisch-römischen Zeit die Niederschrift ganzer Ritualhandbücher beziehungsweise Auszüge davon auf Tempelwänden und Säulen weitaus häufiger als in älterer Zeit. Für die römische Epoche sind die wichtigsten Quellen dabei die Inschriften auf den Säulen des Tempels von Esna. Ihre Hauptmasse stammt aus dem 1. und 2. Jahrhundert $\mathrm{n}$. Chr; dabei liegt der Höhepunkt in der Zeit von Vespasian bis Antoninus Pius. Danach verfällt die Qualität der Inschriften im äußerlichen Bereich zusehends. ${ }^{15}$ Solche aus der Zeit der Severer sind vielfach kaum noch verständlich lesbar, und diejenigen aus der Zeit des Decius verdienen den Namen Hieroglyphen nicht mehr wirklich.

Wichtig ist gerade für die Römerzeit auch die Überlieferung auf Papyri, da aus dieser Zeit erhebliche Mengen von Fragmenten aus Tempelkomplexen erhalten sind, wenngleich diese bislang nur zu einem sehr geringen Teil in Publikationen allgemein zugänglich gemacht worden sind. ${ }^{16}$ Darunter befinden sich auch zahlreiche Fragmente von Ritualhandschriften, die ein reiches und reales Leben der traditionellen ägyptischen Kulte bezeugen und auf eine erhebliche Menge von Personen hinweisen, welche die betreffende Schrift kompetent und in kalligraphisch hochwertiger Form beherrscht haben. In diesen Papyri sind Opfer im Rahmen der Tempelrituale in einem Ausmaß bezeugt, das keinerlei Anlass dafür gibt, irgendeine Art der bewussten Verkleinerung anzunehmen.

Leider ist gerade für die Papyri die Frage der korrekten zeitlichen Einordnung sehr viel schwieriger als für die Monumentalinschriften, da wir kaum ein Kolophon mit genauer Datierung erhalten haben und meist auf paläographische Analysen angewiesen sind - mit allen Un-

${ }^{15}$ S. Sauneron, Quatre campagnes à Esna. Esna I (Kairo 1959), 43-44.

${ }^{16} \mathrm{Vgl}$. als Überblicksartikel insbesondere A. von Lieven, 'Religiöse Texte aus der Tempelbibliothek von Tebtynis - Gattungen und Funktionen', in: S. Lippert M. Schentuleit (Hrsg.), Tebtynis and Soknopaiou Nesos. Leben im römerzeitlichen Ägypten. Akten des Internationalen Symposions, Sommerhausen bei Würzburg 2003 (Wiesbaden 2005), 57-70; K. Ryholt, 'On the content and nature of the Tebtunis temple library. A status report', in: ibid., 141-170; G. Widmer, 'On Egyptian religion at Soknopaiou Nesos in the Roman period (P.Berlin P 6750)', in: ibid., 171-184; J.F. Quack, 'Die hieratischen und hieroglyphischen Papyri aus Tebtynis - ein Überblick', in: Ryholt 2006, a.a.O. (Anm. 6), 1-7. Die Menge der schriftlich tradierten religiösen Texte, die es zu allen Zeiten in Ägypten gegeben hat, lässt jedenfalls die Theorien von Stroumsa 2005, a.a.O. (Anm. 10), 63-101 hinsichtlich der , Religionen des Buches' durchaus etwas problematisch erscheinen. 
sicherheiten, die sich durch einen Mangel an sicher datierten Ankern noch erhöhen. Genau datiert ist vor allem eine Handschrift aus Tebtynis, die exakt auf den 5. September $135 \mathrm{n}$. Chr. festgesetzt ist ${ }^{17}$ allerdings ist dies nicht eine Ritualschrift, sondern eine Beschreibung der lokalen religiösen Landschaft und somit lediglich indirekt als Mittel zur zeitlichen Festlegung paläographisch vergleichbarer Ritualpapyri relevant. Zumindest kann man deutlich sehen, dass diese Papyri vorrangig aus dem 1 . und 2. Jahrhundert $n$. Chr. stammen; ein Ausgreifen in das 3. Jahrhundert ist für diese Fundkomplexe zumindest nicht nachweisbar, und paläographisch sind sie eindeutig früher als die mit gewisser Wahrscheinlichkeit in das dritte Jahrhundert gehörigen magischen Handschriften in demotischer Schrift.

Immerhin hat man - zwar weniger als direkte Ritualhandschriften, doch zumindest als Zeugnisse für den Vollzug eines Kultes mit Opfern - die Narmouthis-Ostraka zur Hand, die uns wertvolle Einblicke in den Alltag der Priester und dabei auch in ihre Tätigkeit im Tempeldienst liefern. ${ }^{18}$ Sie zeigen, dass damals durchaus noch ein Opferkult betrieben wurde. Diese Texte dürften teilweise Vorgänge des späten 2. Jahrhunderts $\mathrm{n}$. Chr. betreffen, zum größten Teil aber (als Niederschriften) aus dem Beginn des 3. Jahrhunderts stammen; jüngster in ihnen belegter Kaiser ist Septimius Severus. ${ }^{19}$ Für das spätere 3. Jahrhundert lassen dann die Quellen für real durchgeführte ägyptische Opfer in Tempeln allerdings drastisch nach. Es sei aber noch darauf hingewiesen, dass in koptischer Sprache im Rahmen des vierten Buches des gnostischen Traktats Pistis Sophia (Kap. 142), die Beschreibung eines Opfers überliefert ist, das zwar vordergründig narrativ als Handlung Jesu und seiner Jünger dargestellt wird, ${ }^{20}$ aber für damalige Leser leicht als Anweisung für ein reales Ritual lesbar gewesen sein dürfte. ${ }^{21}$ Auch dieser Text scheint aus dem 3.

${ }^{17}$ G. Botti, La glorificazione di Sobk e del Fayyum in un papiro ieratico da Tebtynis (Kopenhagen 1959), 73, Taf. IX.

${ }^{18}$ Vgl. insbesondere E. Bresciani - S. Pernigotti - M.C. Betrò, Ostraca demotici da Narmuti I (nn. 1-33) (Pisa 1983); P. Gallo, Ostraca demotici e ieratici dall 'archivio bilingue di Narmouthis II (nn. 34-99) (Pisa 1997); A. Menchetti, Ostraca demotici e bilingui da Narmuthis (ODN 100-188) (Pisa 2005).

19 Menchetti 2005, a.a.O. (Anm. 17), 25.

${ }^{20}$ C. Schmidt, Koptisch-gnostische Schriften, Erster Band. Die Pistis Sophia. Die beiden Bücher des Jeû. Unbekanntes altgnostisches Werk, 3. Auflage bearbeitet von W. Till (Berlin 1962), 243-245.

21 Eine ambitionierte Deutung dieser Stelle versucht R. Eisler, 'Orphischdionysische Mysteriengedanken in der christlichen Antike', in: F. Saxl (Hrsg.), Vor- 
Jahrhundert $\mathrm{n}$. Chr. zu stammen und soll hier nur als Hinweis darauf dienen, wo sich prinzipiell noch weitere Opferbeschreibungen finden lassen könnten.

Insgesamt kann man feststellen, dass es in ägyptischer Sprache in monumentaler hieroglyphischer Form ebenso wie in Ritualhandschriften auf Papyrus eine klar nachzuweisende und ungebrochene Tradition eines Tempelkultes mit Opfern gab, die zumindest bis weit in das 2. Jahrhundert n. Chr. fortexistierte. Für das 3. Jahrhundert lassen dagegen die Quellen deutlich nach. Das 4., eventuell sogar noch das 5. Jahrhundert ist dann die Zeit, aus der die große Masse der von SMITH verwendeten Quellen in Form griechischsprachiger magischer Papyri aus Ägypten stammt. Auch die von SMITH nicht ausgewerteten demotischen magischen Papyri des 3. Jahrhunderts n. Chr. entsprechen den griechischsprachigen hinsichtlich der Art und Dimensionierung der Opfer so gut, dass sein Bild nicht wesentlich beeinträchtigt wird.

Nun könnte man zunächst den Eindruck gewinnen, dass der Begriff der ,Miniaturisierung 'tatsächlich eine brauchbare Beschreibung des geschilderten Vorgangs darstellt: Wir haben ein deutliches $\mathrm{Zu}$ rücktreten der großen Tempelrituale beziehungsweise deren QuasiVerschwinden im Verlauf des 3. Jahrhunderts n. Chr. bemerkt; und wir finden in den magischen Papyri dieser und der nachfolgenden Zeit Rituale, die zum Gutteil auf Traditionen ägyptischer Tempelrituale zurückgehen, aber in kleinerem Maßstab durchgeführt werden. Man braucht das nur als zwei Teile einer linearen Entwicklung zu lesen, und man hätte in der Tat den Befund eines Prozesses der Miniaturisierung, bei dem ursprüngliche Tempelrituale in Aufwand und Dimension verkleinert werden. Allerdings scheint sich mir der Sachverhalt durchaus anders und wesentlich komplexer darzustellen, wenn man den Blick nicht auf die römische Kaiserzeit fixiert, sondern die ägyptische Religion in ihrer langen Geschichte und ihren verschiedenen Praktiken insgesamt betrachtet.

Was von SMITH und ihm folgend von MOYER und DIELEMAN als ,Miniaturisierung' bezeichnet wird, ist tatsächlich alles andere als ein neues Phänomen der Römerzeit. Vielmehr behaupte ich und beabsichtige, es auch durch konkrete Belege nachzuweisen, dass derartige Phänomene schon sehr viel früher Teil des Gesamtspektrums ägypti-

träge der Bibliothek Warburg II. Vorträge 1922-1923, II. Teil (Leipzig - Berlin 1925), 139-158. 
scher Religionsausübung gewesen sind. Als erstes Beispiel möchte ich die Belebung des Ringsteins herausgreifen, welche MOYER und DIELEMAN so eingehend studiert haben. Eine geeignete Parallele hierfür scheint mir im sogenannten Spruch 30B des ägyptischen Totenbuches vorzuliegen. $\mathrm{Zu}$ diesem lautet die Handlungsanweisung in einer Version des Neuen Reiches „Worte sprechen über einem Skarabäus aus Dolerit(?), eingerieben mit Myrrhe. An ihm werde die Mundöffnung vollzogen, er werde in das Herz eines [Mannes] gelegt“. Eine andere Fassung hat „Nun wird ein Skarabäus aus Dolerit(?) hergestellt, gefaßt in Gold, werde in das Herz eines Mannes gegeben, nachdem an ihm die Mundöffnung vollzogen wurde, gesalbt mit Myrrhe“. ${ }^{22}$ Die spätzeitliche Fassung dieses Spruches, welche chronologisch am nächsten zu dem griechischsprachigen Zaubertext steht, lautet: „Worte sprechen über einem Skarabäus aus Dolerit(?), eingerahmt und gereinigt mit Gold, in das Herz eines Mannes legen, nachdem an ihm die Mundöffnung vollzogen wurde. Mit Ö1 bester Qualität salben. ${ }^{623}$ Ich muss gestehen, dass mir der Schritt von einem ägyptischen Herzskarabäus mit seiner Textgravur zu einer magischen Gemme mit Text und Bild nicht so schwerwiegend erscheint, dass hierin ein markanter Prozess bewusster Miniaturisierung gesehen werden müsste.

Hinzu kommt ein weiterer fundamentaler Punkt: Es ist ja keineswegs so, dass bei der Weihe des Ringsteines nur ein miniaturhaftes Modell eines tatsächlichen Objektes verwendet wurde, sondern der Ringstein ist ungeachtet seiner Kleinheit selbst ein voll funktionsfähiges Objekt, das als solches auch in einem klaren zeitgenössischen Bezugsrahmen von Ringsteinen als Amuletten steht. ${ }^{24}$ Auch der Bezug zum traditionellen ägyptischen Mundöffnungsritual, der sich mutmaßlich aus der Etikettierung als Ouphôr ergibt, dürfte in den Details etwas positiver ausfallen, als MOYER und DIELEMAN es angenommen haben. Jedenfalls kann man nicht nur Bezüge zu ägyp-

${ }^{22}$ É. Naville, Das Aegyptische Totenbuch der XVIII. bis XX. Dynastie aus verschiedenen Urkunden zusammengestellt und herausgegeben. Zweiter Band: Varianten (Berlin 1886), 99, Versionen Ih und Pf.

${ }_{23}$ R. Lepsius, Das Todtenbuch der Ägypter nach dem hieroglyphischen Papyrus in Turin mit einem Vorwort zum ersten Male herausgegeben (Berlin 1842), Taf. XVI.

${ }^{24} \mathrm{Zu}$ dieser Gruppe siehe zuletzt zusammenfassend S. Michel, Die magischen Gemmen. Zu Bildern und Zauberformeln auf geschnittenen Steinen der Antike und Neuzeit (Berlin 2004). 
tischer hymnischer Phraseologie im Text erkennen, sondern sogar vergleichsweise enge Verbindungen zu einer spezifischen Szene des Mundöffnungsrituals, wie ich an anderer Stelle nachgewiesen habe. ${ }^{25}$ Das Phänomen an sich, dass das Mundöffnungsritual keineswegs einen normativ verbindlichen Szenenbestand hat, sondern sehr unterschiedliche individuelle Ausprägungen und eine Auswahl aus einer möglichen Langversion aufweist, ist ebenfalls aus viel älterer Zeit bereits gut bekannt und ergibt sich eben daraus, dass das Ritual für verschiedene Objekte und auch von ganz unterschiedlichen gesellschaftlichen Gruppen durchgeführt werden konnte. ${ }^{26}$

Die Vorliebe für kleine Kultstatuen hatte in Ägypten eine lange Tradition, sie muss sogar als grundlegend für den dortigen religiösen Betrieb angesehen werden. Ein wesentlicher Punkt dabei ist, dass im ägyptischen Kult Prozessionsfeste eine große Rolle spielten, folglich die wesentlichen Kultstatuen leicht transportabel sein mussten. ${ }^{27}$ Deshalb ist in etlichen erhaltenen Angaben über die Größe von Kultstatuen eine Höhe von einer Elle, d.h. etwas über $50 \mathrm{~cm}$, so prominent vertreten. ${ }^{28}$ Die Verwendung noch viel kleinerer Objekte als Amulette ist ebenfalls alles andere als neu, sondern zu praktisch allen Zeiten in Ägypten normal gewesen, und stellte zudem wohl kulturübergreifend eine Konstante dar, da Amulette üblicherweise Dimensionen haben sollten, die es problemlos ermöglichten, sie am Körper mit sich zu tragen. ${ }^{29}$

Tatsächlich müsste man, wollte man den Begriff der ,Miniaturisierung' in Ägypten ernstlich angehen, in ganz anderen Zeiten ansetzen, da bereits ab dem Alten Reich insbesondere in Grabbeigaben ein Ersatz von realen und funktionsfähigen Objekten durch Modelle oder

${ }^{25}$ Quack 2006, a.a.O. (Anm. 15), 144-145.

26 J.F. Quack, 'Ein Prätext und seine Realisierungen. Aspekte des ägyptischen Mundöffnungsrituals', in: H. Roeder - B. Dücker (Hrsg.), Text und Ritual. Essays und kulturwissenschaftliche Studien von Sesostris bis zu den Dadaisten (Heidelberg 2005), 165-185.

${ }^{27}$ W. Guglielmi - K. Buroh, 'Die Eingangssprüche des Täglichen Tempelrituals nach dem Papyrus Berlin 3055 (I, 1-VI, 3)', in: J. van Dijk (ed.), Essays on Ancient Egypt in Honour of Herman te Velde (Groningen 1997), 101-166, dort vor allem 102.

${ }^{28}$ F. Hoffmann, Wort und Bild. Texte und Untersuchungen zur ägyptischen Statuenbeschreibung, (Habilitationsschrift Würzburg 2001), 503-512.

${ }^{29} \mathrm{Vgl}$. vorläufig das Material in W.M.F. Petrie, Amulets. Illustrated by the Egyptian Collection in University College, London (London 1914); C. Andrews, Amulets of Ancient Egypt (London 1994); eine neue Monographie zu ägyptischen Amuletten beabsichtige ich selbst zu schreiben. 
Scheingefäße zu beobachten ist. ${ }^{30}$ Man kann sie analytisch mit einer stärkeren intellektuellen Durchdringung verbinden, und ihr reales Auftreten zeigt, dass sie nicht einfach als billigerer Ersatz für das echte Objekt $\mathrm{zu}$ verstehen sind. Allerdings beweist das vor allem einmal mehr, dass es sich hierbei nicht um eine spezifische Entwicklung der Römerzeit handeln kann.

Die Durchführung von Ritualen außerhalb der Tempel sollte ebenfalls nicht als Neuerung angesehen werden. In Ägypten war es schon immer üblich gewesen, private Rituale in Räumen jenseits des Tempels durchzuführen - und das war zunächst auch eine Frage der Zugangsberechtigung innerhalb des Tempels. Dabei handelte es sich nicht einfach nur um Formen der häuslichen Verehrung, ${ }^{31}$ die auch SMITH als ältere Parallelen nennt, sondern es kommen durchaus Ritualdurchführungen in ,freier Natur' vor. Vielleicht kann man als besonders illustratives Beispiel die Durchführung des Rituals zum Totenbuch Kap. 125 heranziehen. ${ }^{32}$ In der Nachschrift zu TB Kap. 125, 5-6 wird angegeben, man solle die Zeichnung auf reinem Boden machen. Neben Ocker wird als Zeichenmaterial auch Ackerboden angegeben, auf den noch kein Schwein oder Kleinvieh getreten ist. Wesentlich an der Durchführung ist einerseits die Reinheitsfrage, bei der bestimmte Tiere als unrein betrachtet werden. Daneben spielt auch eine Rolle, dass es sich um eine Fläche handelt, die sozusagen noch unmarkiert und durch niemand anderen verwendet ist. Strukturell entspricht dies gut dem Vorgehen etwa im griechischsprachigen Material PGM IV 27-30, wo als Platz der rituellen Weihe ein Ort vorgeschrieben wird, der erst kürzlich vom Nil gesäubert wurde und dessen Spülkreis noch niemand betreten hat. Ebenso vergleichbar ist in einem demotischen magischen Papyrus, dass reiner Sand vom Fluß hergebracht wird, um den Boden eines Raums zu bestreuen, in dem eine magische Handlung stattfindet (PMag. LL 10, 10).

${ }^{30}$ R. Schiestl, Modellgefäße der prä- und frühdynastischen Zeit in Ägypten, (Magisterarbeit Wien 1996); S. Allen, 'Miniature and model vessels in ancient Egypt', in: M. Bárta (ed.), The Old Kingdom: Art and Architecture. Proceedings of the Conference held in Prague 2004 (Prag 2006), 19-24.

${ }^{31}$ Vgl. für Ägypten etwa A.I. Sadek, Popular Religion in Egypt during the New Kingdom (Hildesheim 1987); G. Bomann, The Private Chapel in Ancient Egypt. A Study of the Chapels in the Workmen's Village at El Amarna with special reference to Deir el Medina and other Sites (London - New York 1991); A. Stevens, Private Religion at Amarna. The Material Evidence (Oxford 2006).

${ }_{32}$ Naville 1886, a.a.O. (Anm. 21), 332-334. 
Zudem ist die schwerpunktmäßige Verwendung der eigenen häuslichen Räume als Ort des Rituals in den magischen Papyri sicher auch wesentlich durch einen ganz elementaren Punkt bedingt: Magie war im römischen Reich von staatlicher Seite verboten, und hierauf standen schwere Strafen. Sehr viele der magischen Praktiken in den Zauberpapyri waren ferner - ganz unabhängig von der aktuellen Gesetzgebung - in einem solchen Maße gesellschaftlich zwielichtig oder eindeutig auf die Schädigung anderer Personen ausgerichtet, dass elementarste Vorsicht dazu geraten haben dürfte, sie den Blikken anderer Menschen zu entziehen. Als weiterer Punkt ist zu beachten, dass viele der Rituale, insbesondere solche, in denen es um die Erzielung von Visionen ging, in ihrer praktischen Durchführung stark von einer absolut ungestörten Durchführung abhingen, welche die Konzentration des Ritualisten ungebrochen halten konnte. ${ }^{33}$ Hierfür eigneten sich naturgemäß private Räume erheblich besser.

Weiterhin zu erörtern ist die Frage der kleinen Opfer auf kleinen Altären. Auch hierin vermag ich nichts in irgendeiner Form wirklich Neues zu erkennen. Sinnvoll dürfte es sein, hier einmal Belege aus erheblich älterer Zeit in Ägypten zu betrachten, die bereits strukturell gleichartige Handlungen zeigen. ${ }^{34}$ Grundsätzlich war es in Ägypten nichts Ungewöhnliches, wenn ein Opfer im Rahmen einer privaten Götterverehrung oder eines magischen Aktes sich auf wenige Opfergaben von bescheidenem materiellem Aufwand beschränkte. Einige Beispiele sollen dies illustrieren: In vielen Fällen von Heilzaubern gab es überhaupt keine dezidierten Opfer, sondern lediglich eine Verwendung an sich offizinaler Substanzen in Verbindung mit der Rezitation von Beschwörungen. In anderen Fällen wurden magische Zeichnungen angefertigt und (meist am Hals) dem Patienten angelegt. Dies kann gelegentlich (zum Beispiel PChesterBeatty VIII vs. $10,1)^{35}$ von einer Räucherung begleitet werden, sehr oft ist aber in ägyptischen magischen Papyri zu allen Zeiten die Rezitation allein in Verbindung mit einer Zeichnung und/oder Niederschrift des Beschwörungstextes durchgeführt worden. Damit habe ich auch bereits einen weiteren Punkt in SMITHs Theorie aufgegriffen, nämlich den

${ }^{33}$ Genauer werde ich dies in J.F. Quack, 'Postulated and real efficacy in late antique divination rituals', Journal of Ritual Studies (im Druck) ausführen.

${ }^{34}$ Vgl. hier das bei P. Eschweiler, Bildzauber im alten Agypten (Freiburg - Göttingen 1994), 256-257 zusammengestellte Material.

${ }^{35}$ A.H. Gardiner, Hieratic Papyri in the British Museum, Third Series. Chester Beatty Gift (London 1935), 75, Taf. 47. 
Ersatz der rituellen Handlung durch den Akt des Schreibens als solchen. Wenn man bedenkt, wie sehr schon in älteren ägyptischen magischen Texten die reine Textrezitation samt Zeichnung beziehungsweise Niederschrift im Zentrum steht, ja der einzige konkrete Handlungspunkt sein kann, ${ }^{36}$ sehe ich auch hier nicht, was in den griechischsprachigen Papyri der Römerzeit wesentlich neu sein soll.

Andere Fälle zeigen konkrete Opfer, aber diese sind in der Mehrzahl der Fälle von einem materiellen Zuschnitt, der sich nicht signifikant von demjenigen der Papyri Graecae Magicae unterscheidet. Generell dürften hier Totenbuchsprüche ein besonders gutes Vergleichsmaterial liefern, da sie privat durchgeführte Rituale außerhalb des Tempelkultes überliefern, einige von ihnen recht ausführliche Verwendungsanweisungen enthalten und ihr Belegzeitraum ab dem Neuen Reich, also der zweiten Hälfte des 2. Jahrtausends v. Chr., so weit vor den hier zu diskutierenden magischen Papyri liegt, dass sich die Frage einer chronologisch relevanten Entwicklung gut testen lässt. Die Nachschrift zu TB 130 gibt an, man solle an einem reinen Ort operieren. Vor einer magischen Zeichnung mit einem Abbild des Verstorbenen werden Brot, Bier und ,alle guten Dinge“ geopfert. Zu $T B 133$ werden überhaupt nur eine magische Zeichnung sowie rituelle Reinheit vorgeschrieben, Opfer dagegen finden überhaupt keine Erwähnung. Im Falle von TB 134 werden erneut magische Zeichnungen (auf einer Schale) angefertigt, dazu werden Weihrauch und gebratene Vögel geopfert.

Ein vergleichsweise aufwendiges Opfer stellt es schon dar, wenn im Heilzauber des PChesterBeatty V, vs. 7, 3-5 sieben Portionen gerösteter Ziegenleber verwendet werden ${ }^{37}$ - doch auch diese konnte man im Notfall mutmaßlich aus einer einzigen Ziegenleber gewinnen, je nachdem wie groß oder klein die Portionsschalen waren. Ein umfangreicheres Opferarrangement bietet dagegen PChester Beatty VIII rt. 4, 3-7. ${ }^{38}$ Dort gibt es sieben Weißbrote, sieben Grillstücke, sieben Rippchen, sieben Menschen aus Weißbrot, sieben Gurken, [...], vier Schalen [...], vier Schalen Salz, vier Portionen geröstetes Fleisch, vier Schalen Milch, vier Schalen Weihrauch, vier Schalen

36 Genügend Textbeispiele finden sich in J.F. Borghouts, Ancient Egyptian Magical Texts (Leiden 1978).

${ }_{37}$ Gardiner 1935, a.a.O. (Anm. 34), 52, Taf. 29.

${ }^{38}$ Gardiner 1935, a.a.O. (Anm. 34), 68, Taf. 40. Die Liste der Opfergaben ist leider nur fragmentarisch erhalten. 
guten Wein, [...] vier Krüge Bier, vier Körbe Gemüse. Weitere Ingredienzien, besonders einige lebende Tiere, scheinen keine Opfer an die Götter zu sein, sondern zu Substitutionszwecken eingesetzt zu werden. Von den Angaben eines anderen Spruches derselben Handschrift sind nur noch sieben Männer aus Brot, sieben Herzen und sieben Vögel erhalten (PChesterBeatty VIII rt. 11, 4) (9) $^{39}$ hier mag man sich an die sieben verschiedenen Vögel in PGM XX 30-31 erinnern.

Ausnahmsweise relativ aufwendig ist nur die Nachschrift zu $T B$ 144, wo mit Rinderschenkeln, Herz und Rippenstücken wenigstens der Einsatz eines ganzen Rindes (neben Broten, Bier, Milch, Kräutern, Ölen, Schminke und Weihrauch) notwendig wird. ${ }^{40}$ Allerdings gibt es gute Gründe zur Annahme, dass dieser Totenbuchspruch, in dem es um das Eintreten durch sieben Tore zum Bereich des Osiris geht, ursprünglich auf eine Tempelkulttradition eben im Ritual für Osiris zurückgeht und somit Dimensionierungen der Opfer zeigt, die für staatliche Kulte intendiert und nicht auf einfache Privatpersonen zugeschnitten waren. ${ }^{41}$ Zudem findet sich die lange Liste der nötigen Opferbestandteile keineswegs in allen Handschriften des Spruches; im Neuen Reich fehlt sie meist.

Zusammengenommen kann eine Tendenz zur Verkleinerung der im Ritual verwendeten Objekte und der Opfer in Ägypten keinesfalls als Spezifikum der Römerzeit beziehungsweise der griechischsprachigen magischen Papyri angesehen werden. Sie ergibt sich vielmehr weitgehend aus den ökonomischen Gegebenheiten, ohne dass es notwendig, ja überhaupt sinnvoll erscheint, in sie so etwas wie ein ,Ritual des Rituals' mit entsprechenden intellektuellen Hintergründen hineinzuinterpretieren. Tatsächlich ist allenthalben nachweisbar, dass ähnliche Phänomene einer materiellen Bescheidenheit und Unabhängigkeit vom definierten Kultort des Tempels in Ägypten zu allen Zeiten $\mathrm{zu}$ fassen sind, sobald man sich aus dem öffentlichen Rahmen entfernt und Rituale für Privatpersonen untersucht. Ein Vergleich der griechischsprachigen magischen Papyri mit den ägypti-

\footnotetext{
${ }^{39}$ Gardiner 1935, a.a.O. (Anm. 34), 71, Taf. 43.

${ }^{40} \mathrm{G}$. Lapp, Catalogue of the Books of the Dead in the British Museum, Volume I. The Papyrus of $\mathrm{Nu}$ (London 1997), Taf. 76.

${ }^{41}$ Vgl. A. von Lieven, 'Book of the Dead, Book of the Living', in: S. Seidlmayer (ed.), Religion in Context (im Druck). Aus diesem Grund habe ich in der Diskussion auch keine Unterweltsbücher ausgewertet, da diese in den ersten belegten Überlieferungszusammenhängen exklusiv für den König genutzt wurden und somit die materiellen Möglichkeiten ganz andere waren.
} 
schen Tempelritualen dürfte in diesem Bereich schon deshalb heikel sein, weil die zugrundeliegenden materiellen Fakten einfach andere waren. Dennoch gibt es einen letzten Punkt, mit dem ich auch endlich die Frage der Auswirkungen des römischen Reiches etwas detaillierter aufgreifen möchte, und dieser betrifft eben die materielle Basis.

Früher lautete die die gängige Theorie, dass der römische Staat den reichen Grundbesitz, der für die ägyptischen Tempel noch in der Ptolemäerzeit eine wesentliche Quelle ihres Reichtums darstellte, einfach eingezogen habe. Dies ist zwar inzwischen durch neuere Quellen widerlegt, die definitiv auch in der Römerzeit noch heiliges Land als Grundbesitz zeigen, ${ }^{42}$ aber dennoch dürfte unbestritten sein, dass die ökonomischen Möglichkeiten der ägyptischen Tempel unter der Römerherrschaft deutlich eingeschränkt waren - dies ist auch an der merklich nachlassenden Intensität des Tempelbaus abzulesen. ${ }^{43}$

Und gerade auf dieser Grundlage kann man wohl der Frage nachgehen, inwieweit es in Ägypten einen ,Impact of Empire' auf die traditionellen Kulte gegeben hat. Ich glaube, dass es keinen Einfluß in dem Sinne gegeben hat, dass die römische Politik explizit Änderungen der Rituale herbeiführen wollte. Gerade dadurch, dass die römische Eroberung Ägyptens und die Strukturierung des römischen Reiches als Monarchie fast zusammenfielen, war eine Integration des römischen Kaisers in die alten Opferkulte in der Rolle des Pharaos problemlos möglich. Auswirkungen gab es allenfalls indirekt durch die nunmehr knapperen finanziellen Mittel, was sich daraus ergab, dass Rom aus dieser Provinz einen Überschuss erwirtschaften wollte. In dem Maße, wie im Zusammenhang der Reichskrisen und des ökonomischen Niederganges im 3. Jahrhundert die ägyptischen Tempel von Finanznot betroffen waren, gehen auch die Quellen für Opferkulte in Gestalt von dekorierten Tempelwänden oder Ritualhandschriften so stark zurück, dass sie unter Einrechnung des Faktors der Erhaltung für uns kaum noch fassbar sind. Dabei muss es sich keineswegs um Krisen und Probleme innerhalb Ägyptens selbst gehandelt haben, sondern lediglich um solche, deren Folgen für den Gesamthaushalt des Römischen Reiches einschneidend genug waren,

\footnotetext{
42 A. Monson, 'Sacred land in Ptolemaic and Roman Tebtunis', in: Lippert Schentuleit 2005, a.a.O. (Anm. 15), 79-91.

${ }^{43}$ Vgl. hier etwa G. Hölbl, Altägypten im Römischen Reich. Der römische Pharao und seine Tempel I-III (Mainz 2000-05).
} 
um zu einer Kürzung der innerhalb Ägyptens verfügbaren Mittel zu führen. Außerdem ist zu beachten, dass in Ägypten vor der römischen Eroberung eine staatliche Kultfinanzierung in einem Außmaß betrieben wurde, wie es dem Zuschnitt keiner anderen römischen Provinz entsprach, und die römische Reichsverwaltung wenig Interesse daran gehabt haben wird, die Dinge dauerhaft auf diesem Niveau aufrechtzuerhalten.

Damit werden die griechischsprachigen magischen Papyri ab dem 3. Jahrhundert n. Chr. fast zu unserer einzigen Quelle für Rituale in Ägypten. Die in ihnen fassbaren, geringen Ausmaße von Objekten und Opfergaben sind aber keineswegs eine Innovation im Sinne eines bewusst überlegten neuen Konzepts von ,Miniaturisierung6, sondern jahrtausendealter Normalfall; lediglich das Verschwinden der vorher daneben stets präsenten, groß angelegten staatlichen Opfer ist neu. $\mathrm{Zu}$ fragen wäre allerdings, welche Auswirkungen es auf die generelle Religiosität gehabt haben mag, wenn die bisher vorhandenen staatlichen Kulte abnahmen oder gar gänzlich verschwanden. Eine solche Frage müsste freilich anders ansetzen, denn die von Individuen für sehr spezifische Bedürfnisse durchgeführten Rituale, wie sie in den gräkoägyptischen magischen Papyri zu fassen sind, haben wenig Relevanz hinsichtlich des religiösen Lebens der Gemeinschaften im Ganzen. Insofern würde sie ein ganz neues Kapitel eröffnen, von dessen Behandlung an dieser Stelle abgesehen sei.

Heidelberg, Februar 2008 